Pacific Journal of Mathematics

THE AMALGAMATION PROPERTY IN EQUATIONAL 


\title{
THE AMALGAMATION PROPERTY IN EQUATIONAL CLASSES OF MODULAR LATTICES
}

\author{
G. GRÄTZer, B. JóNSSON, AND H. LAKSER*
}

It is known that the class of all distributive lattices satisfies the Amalgamation Property. It will be shown that this is the only nontrivial equational class of modular lattices for which the Amalgamation Property holds. A second theorem gives further information about the Amalgamation Class of the class of all modular lattices, and of certain other equational classes.

1. Introduction. A class $K$ of algebras (or in general, structures) is said to have the Amalgamation Property if for $A, B_{0}, B_{1}$ in $\boldsymbol{K}$ and for embeddings $f_{i}: A \rightarrow B_{i}, i=0,1$, there exist a $C$ in $\boldsymbol{K}$ and embeddings $g_{i}: B_{i} \rightarrow C, i=1,2$, such that $f_{0} g_{0}=f_{1} g_{1}$ (see Figure 1).

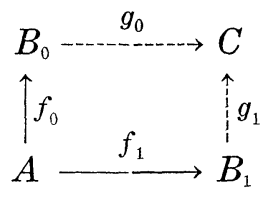

FIGURE 1.

For the history and importance of the Amalgamation Property we refer the reader to B. Jónsson [8] (see also G. Grätzer [5]).

The class of all lattices and the class of all distributive lattices both have the Amalgamation Property. The problem whether the class $\boldsymbol{M}$ of all modular lattices has the Amalgamation Property has been around for more than a decade.

In January of 1971 B. Jónsson announced [11] that $\boldsymbol{M}$ does not have the Amalgamation Property, in fact, any equational class $\boldsymbol{K}$ of modular lattices having the Amalgamation Property must satisfy the arguesian identity. This was followed by an announcement by G. Grätzer and H. Lakser [7] stating that every member of $\boldsymbol{K}$ can be embedded into the subspace lattice of an infinite dimensional projective geometry. Combining and extending these results, we can now prove the following:

THEOREM 1. If an equational class $\boldsymbol{K}$ of modular lattices contains a nondistributive lattice, then $\boldsymbol{K}$ does not have the Amalgamation Property.

If $K$ does not have the Amalgamation Property, then we can use the concept of the Amalgamation Class of $\boldsymbol{K}$ (G. Grätzer and H. Lakser 
[6]) to measure to what extent the Amalgamation Property holds. We define $A$ to be in the Amalgamation Class of $\boldsymbol{K}(A \in \operatorname{Amal}(\boldsymbol{K}))$ if and only if for any $B_{0}, B_{1} \in \boldsymbol{K}$ and embeddings $f_{i}: A \rightarrow B_{i}, i=0,1$, there exist a $C \in \boldsymbol{K}$ and embeddings $g_{i}: B_{i} \rightarrow C, i=0,1$, such that $f_{0} g_{0}=f_{1} g_{1}$. If $\boldsymbol{K}$ is an equational class of lattices, then it is easily seen that the one-element lattice is always in Amal $(\boldsymbol{K})$.

THEOREM 2. No distributive lattice with more than one element belongs to Amal (M). In fact, if $\boldsymbol{K}$ is any nontrivial equational class of modular lattices and Amal $(\boldsymbol{K})$ contains a distributive lattice with more than one element, then $\boldsymbol{K}$ satisfies the arguesian identity.

Some steps in the proofs of Theorems 1 and 2 rely on ideas of R. P. Dilworth and M. Hall [1] even though no result of [1] could be applied directly. The proof of Theorem 1 makes heavy use of some deep results on projective geometries. The proof of Theorem 2 is patterned after the proof of Desargues' theorem for projective geometries of dimension 3 or more.

Theorems 1 and 2 are proved in Sections 2 and 3, respectively. The fourth and final section contains some comments and problems and a result to the effect that a variety generated by a finite nondistributive lattice never has the Amalgamation Property.

2. Proof of Theorem 1. In this section let $\boldsymbol{K}$ be an equational class of modular lattices that contains a nondistributive lattice and has the Amalgamation Property. We are going to prove a series of statements about $\boldsymbol{K}$ that will lead to a contradiction, and thus to a proof of Theorem 1 .

$\Lambda$ diamond is the five element modular nondistributive lattice of Figure 2. That the diamond belongs to $\boldsymbol{K}$ follows from the assumptions.

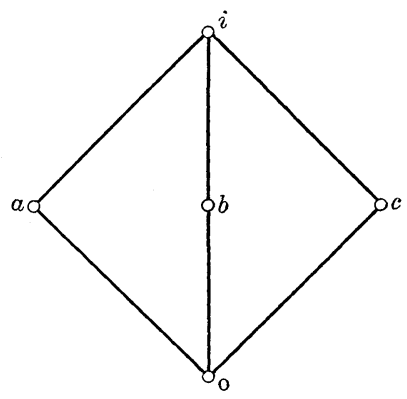

FIGURE 2.

Statement 1. Every member of $\boldsymbol{K}$ can be embedded in a simple 
complemented lattice that also belongs to $\boldsymbol{K}$.

Proof. Let $L$ be a lattice in $\boldsymbol{K}$. We can assume that $L$ has 0 and 1 because if a lattice belongs to $\boldsymbol{K}$ then that lattice with 0 and 1 adjoined also belongs to $\boldsymbol{K}$. Consider a fixed diamond labelled as in Figure 2. Given $x \in L, x \neq 0,1$, we can take the embeddings

$$
\begin{aligned}
& f_{0}: o \rightarrow 0, a \rightarrow x, i \rightarrow 1 \\
& f_{1}: o \rightarrow o, a \rightarrow a, i \rightarrow i
\end{aligned}
$$

of $\{0, a, i\}$ into $L$ and into the diamond, respectively. By the Amalgamation Property there is a lattice $L_{1}$ in $K$ containing $L$ as a sublattice having the same 0 and 1 as $L$, such that $\{0, x, 1\}$ is contained in a diamond sublattice of $L$. Iterating this process, possibly a transfinite number of times (this is possible because $\boldsymbol{K}$ is closed under direct limits), we obtain a lattice $N$ that contains $L$ as a sublattice, has the same 0 and 1 as $L$, and has the property that for every element $x \in L, x \neq 0,1$, there exist $y, z \in N$ such that $\{0, x, y, z, 1\}$ is a diamond.

Repeating this process we obtain an infinite sequence

$$
L=C_{0} \leqq C_{1} \subseteq \cdots \sqsubseteq C_{n} \subseteq C_{n+1} \leqq \cdots
$$

of lattices in $K$, all having the same 0 and 1 as $L$, and having the property that, for all $n<\omega$, every element $x$ of $C_{n}, x \neq 0,1$, belongs to a diamond $\{0, x, y, z, 1\}$ in $C_{n+1}$.

The union $C_{\infty}$ of the $C_{n}$ belongs to $\boldsymbol{K}$ and it is obviously complemented. To show that $C_{\infty}$ is simple, recall that in a complemented modular lattice every congruence $\Theta$ is determined by the ideal [0] $\Theta$ of elements congruent to 0 modulo $\Theta$. If $\Theta \neq \omega$ (the trivial congruence), then there exists an $x \in C_{\infty}, x \neq 0$, such that $x \equiv 0(\Theta)$. If $x=1$, then $\Theta=\iota$ (the largest congruence). If $x<1$, then for some $n<\omega$, we have $x \in C_{n}$ and so there exists a diamond $\{0, x, y, z, 1\}$ in $C_{n+1}$. Since a diamond is a simple lattice, it follows that $0 \equiv 1(\Theta)$, so that $\Theta=\iota$ in $C_{\infty}$. This shows that $C_{\infty}$ is a simple lattice, completing the proof of Statement 1 .

If $P$ is a projective geometry, let $\mathscr{C}(P)$ denote the lattice of all subspaces of $P$. We say that $P$ is degenerate if some line contains only two points; otherwise $P$ is called nondegenerate.

Statement 2. For every member $L$ of $\boldsymbol{K}$ there exists an infinite dimensional nondegenerate projective geometry $P$ such that $L$ can be embedded in $\mathscr{P}(P)$ and $\mathscr{P}(P)$ belongs to $\boldsymbol{K}$.

Proof. We may assume that $L$ has 0 and 1 and that it contains 
an infinite chain, because if it does not, adjoin an infinite chain to "the top of" $L$ and the resulting lattice is still in $K$. By Statement 1, $L$ can be embedded in a simple complemented lattice $C$ of $K$. By O. Frink [4] $C$ can embedded into some $\mathscr{L}(P)$ where $P$ is a projective geometry; crucial for our argument is the result of B. Jónsson [9] according to which $P$ can be chosen so that $\mathscr{L}(P) \in K$. The lattice $\mathscr{L}(P)$ has a representation

$$
\mathscr{L}(P) \cong \Pi\left(\mathscr{L}\left(P_{i}\right) \mid i \in I\right)
$$

where the $P_{i}, i \in I$, are nondegenerate projective geometries. Let $\varphi_{i}$ denote the embedding of $C$ into $\mathscr{L}(P)$ followed by the $i$ th projection. Since $\varphi_{i}$ preserves 0 and 1 , it cannot be a constant homomorphism. Thus, since $C$ is simple, $\varphi_{i}$ is an embedding of $C$ into $\mathscr{L}\left(P_{i}\right)$. Since $C$ contains an infinite chain, $P_{i}$ is infinite dimensional, completing the proof of Statement 2.

Let $D$ be a division ring and let $V$ be a vector space over $D$. Let $\mathscr{L}(V, D)$ denote the lattice of all subspaces of $V$. We define a projective space $P$ as follows: the points of $P$ are the onedimensional subspaces of $V$; if $A$ and $B$ are distinct one-dimensional subspaces of $V$, then the line $A+B$ through $A$ and $B$ consists of all one-dimensional subspaces $C$ that are contained in the subspace spanned by $A$ and $B$. Then $P$ is a nondegenerate projective space and

$$
\mathscr{L}(V, D) \cong \mathscr{L}(P) \text {. }
$$

The celebrated coordinatization theorem of projective geometry asserts that if $P$ is a nondegenerate projective geometry and if $\mathscr{L}(P)$ contains a five element chain, then there exist a division ring $D$ and a vector space $V$ over $D$ such that

$$
\mathscr{L}(P) \cong \mathscr{L}(V, D) \text {. }
$$

This division ring, called the coordinate ring of $P$, is unique up to isomorphism. All the lines of $P$ have the same number of points: this number is $|D|$ if $D$ is infinite and $|\mathrm{D}|+1$ if $D$ is finite.

Statement 3. There exists a projective plane $Q$ such that $\mathscr{L}(Q)$ belongs to $K$ and $Q$ has at least six points on each line.

Proof. By Statement 2, there exist an infinite dimensional nondegenerate projective geometry $P$ such that $\mathscr{L}(P)$ belongs to $K$. If $P$ has the property that every line has at least six points, then choose as $Q$ any plane in $P$. Since $\mathscr{L}(Q)$ is a sublattice of $\mathscr{L}(P)$, we conclude that $\mathscr{L}(Q) \in \boldsymbol{K}$ and, obviously, every line of $Q$ has at 
least six points. If the lines in $P$ have less than six points, then the coordinate ring of $P$ must be a field $F$ of order 2 , 3, or 4 . Therefore,

$$
\mathscr{L}(P) \cong \mathscr{L}(V, F)
$$

for some infinite dimensional vector space $V$ over $F$. Let us choose a finite field extension $K$ of $F$ of order $k \geqq 5$, and let $W$ be a three-dimensional vector space over $K$. Then $\mathscr{L}(W, K)$ determines a projective plane $Q$ (in the sense that $\mathscr{L}(W, K) \cong \mathscr{L}(Q)$ ).

$\mathscr{L}(W, K)$ is a sublattice of $\mathscr{L}(W, F)$, and $\mathscr{L}(W, F)$ is isomorphic to a sublattice of $\mathscr{L}(V, F)$, proving that $\mathscr{L}(Q)$ is in $\boldsymbol{K}$. Since every line in $Q$ has at least $k+1 \geqq 6$ points, this completes the proof of Statement 3.

The next two statements contain known facts that apply to any class that has the Amalgamation Property.

Statement 4. Let $C, D \in \boldsymbol{K}$. Let $D$ be an extension of $C$ and let $\varphi: C \rightarrow D$ be a one-to-one homomorphism. Then there exists an extension $E$ of $D(E \in \boldsymbol{K})$ and a one-to-one homomorphism $\beta: D \rightarrow E$ extending $\varphi$.

Proof. $C \subseteq D$ and $\varphi: C \rightarrow D$; hence we can apply the Amalgamation Property to get an extension $E$ of $D$ and a one-to-one homomorphism $\beta: D \rightarrow E$ such that for $c \in C$ we have $c \varphi=c \beta$, completing the proof.

Statement 5. Let $A, B \in \boldsymbol{K}$. Let $B$ be an extension of $A$ and let $\alpha$ be an automorphism of $A$. Then there exists an extension $\bar{B} \in \boldsymbol{K}$ of $B$ and an automorphism $\bar{\alpha}$ of $\bar{B}$ extending $\alpha$.

Proof. We define (see Figure 3) a sequence of extensions of $A$ :

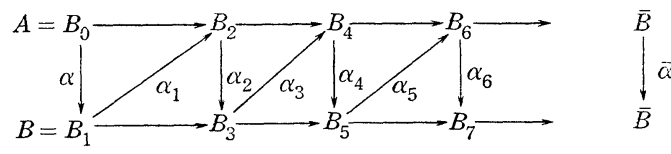

FIGURE 3.

$$
A=B_{0} \subseteq B=B_{1} \subseteq B_{2} \subseteq \cdots \leqq B_{n} \subseteq B_{n+1} \subseteq \cdots
$$

and a sequence of one-to-one homomorphisms $\alpha_{n}: B_{n} \rightarrow B_{n+1}$ such that $\alpha_{0}=\alpha$ and $\alpha_{n+1}$ extends $\alpha_{n}^{-1}$ (on $\operatorname{Im}\left(\alpha_{n}\right)$ ) for all $n$. Indeed, if we have $B_{n}, B_{n+1}$ and $\alpha_{n}$, we apply Statement 4 to $C=\operatorname{Im}\left(\alpha_{n}\right)$, $D=B_{n+1}, \varphi=\alpha_{n}^{-1}$ and we get $B_{n+2}$ an extension of $B_{n+1}$ and $\alpha_{n+1}$ : $B_{n+1} \rightarrow B_{n+2}$ extending $\alpha_{n}^{-1}: \operatorname{Im}\left(\alpha_{n}\right) \rightarrow B_{n+1}$. We then define 


$$
\bar{B}=\bigcup\left(B_{n} \mid n<\omega\right), \bar{\alpha}=\bigcup\left(\alpha_{2 n} \mid n<\omega\right) .
$$

Obviously $\bar{\alpha}$ is a one-to-one homomorphism. Let $y \in \bar{B}$. Then there is an $n$ such that $y \in B_{2 n+1}$. Let $x=y \alpha_{2 n+1}$; since $\alpha_{2 n+2}$ is an extension of $\alpha_{2 n+1}^{-1}, x \bar{\alpha}=x \alpha_{2 n+2}=y$. Hence $\bar{\alpha}$ is an automorphism of $\bar{B}$ extending $\alpha$, completing the proof of Statement 5 .

We are now ready to start the final argument leading to the desired contradiction.

By Statement 3 there exists a projective plane $Q$ with at least six points on each line that $\mathscr{L}(Q) \in K$. By Statement 2 there exists an infinite dimensional nondegenerate projective geometry $P$ such that $\mathscr{L}(P) \in \boldsymbol{K}$ and $\mathscr{L}(Q)$ is isomorphic to a sublattice of $\mathscr{L}(P)$. By the coordinatization theorem, there exists a vector space $V$ over a division ring $D$ such that $\mathscr{L}(P) \cong \mathscr{L}(V, D)$. Consequently there exists a one-to-one homomorphism of $\mathscr{L}(Q)$ into $\mathscr{L}(V, D)$.

The fact that $\mathscr{L}(Q)$ is isomorphic to a sublattice of the lattice of all subgroups of an abelian group,- the group $V$,-implies that the arguesian identity holds in $Q$. Therefore coordinates can be introduced in $Q$ in the usual manner, and we shall actually have to make use of part of that construction. Choose a line $m$ in $Q$ and two distinct points $a_{0}$ and $a_{\infty}$ on $m$, and let $K=m-\left\{a_{\infty}\right\}$. Choose two distinct points $p$ and $q$ of $Q$ that are collinear with $a_{0}$ but are not on $m$. Given $x, y \in K$, let

$$
\begin{aligned}
u & =(x \vee p) \wedge\left(q \vee a_{\infty}\right), v=(y \vee q) \wedge\left(p \vee a_{\infty}\right), \\
x+y & =(u \vee v) \wedge m .
\end{aligned}
$$

$K$ is of course an abelian group under this operation, and the operation is independent of the points $p$ and $q$.

Any permutation of the points of $m$ induces an automorphism of the interval $[0, m]$. Using the fact that $m$ has at least six points, we can choose $x, y \in K-\left\{a_{0}\right\}$ so that $x+y \neq a_{0}$, and we can then find an automorphism $\alpha$ of the interval $[0, m]$ that keeps $a_{0}, a_{\infty}, x$ and $y$ fixed but maps $x+y$ onto a point $z \neq x+y$. By Statement 5 there exists an extension $L$ of $\mathscr{L}(Q)$ such that $\alpha$ extends to an automorphism $\beta$ of $L$ and, as above, by Statement 2 there exists an embedding $F$ of $L$ into the lattice of all subgroups of an abelian group $A$. We claim that $F(x+y)$ is the set of all elements $a \in A$ such that, for some $b, c \in A$

$$
b \in F(x), c \in F(y), a-b \in F\left(a_{\infty}\right), a-c \in F\left(a_{\infty}\right), a-b-c \in F\left(a_{0}\right) .
$$

Let $u$ and $v$ be as in (1). First assume that $a \in F(x+y)$. Since $x+y \leqq u \vee v$, we have $F(x+y) \subseteq F(u \vee v)=F(u)+F(v)$, and so there exists a $d \in A$ satisfying 


$$
d \in F(u), a-d \in F(v) .
$$

Since $u \leqq x \vee p$ and $v \leqq y \vee q$, it follows that there exist $b, c \in A$ such that

$$
b \in F(x), d-b \in F(p), c \in F(y), a-d-c \in F(q) .
$$

The element $a-b$ belongs to each of the groups $F(x+y)+F(x)$ and $F(v)+F(p)$. Since

$$
((x+y) \vee x) \wedge(v \vee p) \leqq m \wedge(v \vee p) \leqq a_{\infty},
$$

it follows that $a-b \in F\left(a_{\infty}\right)$. Similarly, $a-c \in F\left(a_{\infty}\right)$ and $a-b-c \in$ $F\left(a_{0}\right)$, verifying (3).

Conversely, assume that (3) holds. Since $a_{0} \leqq p \vee q$, there exists an $n \in A$ such that

$$
n \in F(p), a-b-c-n \in F^{\prime}(q) .
$$

Using (1) we infer that

$$
b+c \in F(u) \text { and } a-b-c \in F(v),
$$

and so (2) implies that $a \in F(x+y)$.

In this argument we can replace $F(t)$ by

$$
F^{\prime}(t)=F(t \beta) \text {. }
$$

Since $F$ and $F^{\prime}$ agree on $a_{0}, a_{\infty}, x$, and $y$, it follows from the description (3) that

$$
F(x+y)=F^{\prime}(x+y) \text {. }
$$

This, however, is impossible since $F^{\prime}(x+y)=F(z)$ and $z \neq x+y$. This contradiction proves the theorem.

It should be observed that if we only want to show that $\boldsymbol{M}$ fails to have the Amalgamation Property, then it suffices to combine Statement 1 with the example of R. P. Dilworth and M. Hall [1] of a modular lattice that cannot be embedded in a complemented modular lattice.

A naive first approach to Theorem 1 would be to find three lattices $A, B_{0}, B_{1}, A$ a sublattice of $B_{0}$ and $B_{1}$, in the equational class generated by the diamond that cannot be amalgamated in the class of all modular lattices. However, it follows from results in [3] that such an amalgamation can always be effected.

3. Proof of Theorem 2. In preparation for the proof of Theorem 2, we establish three lemmas that are of some independent interest. 
We define a triangle in a lattice $L$ to be an ordered triple of elements of $L$. We shall have frequent occasions to consider two triangles in $L, a=\left\langle a_{0}, a_{1}, a_{2}\right\rangle$ and $b=\left\langle b_{0}, b_{1}, b_{2}\right\rangle$, and the following associated elements:

$$
\begin{aligned}
& p=\left(a_{0} \vee b_{0}\right) \wedge\left(a_{1} \vee b_{1}\right) \wedge\left(a_{2} \vee b_{2}\right), \\
& c_{0}=\left(a_{1} \vee a_{2}\right) \wedge\left(b_{1} \vee b_{2}\right), \quad c_{1}=\left(a_{2} \vee a_{0}\right) \wedge\left(b_{2} \vee b_{0}\right), \\
& c_{2}=\left(a_{0} \vee a_{1}\right) \wedge\left(b_{0} \vee b_{1}\right), \quad m=c_{2} \wedge\left(c_{0} \vee c_{1}\right) .
\end{aligned}
$$

The symbols $p, c_{0}, c_{1}, c_{2}$ and $m$ will always be used in this sense.

Two triangles $a$ and $b$ in $L$ are said to be centrally perspective provided $p=\left(a_{0} \vee b_{0}\right) \wedge\left(a_{1} \vee b_{1}\right)$ or, equivalently,

$$
\left(a_{0} \vee b_{0}\right) \wedge\left(a_{1} \vee b_{1}\right) \leqq a_{2} \vee b_{2},
$$

and $p$ is then called the center of perspectivity of $a$ and $b$. The triangles $a$ and $b$ are said to be axially perspective provided $m=c_{2}$, or, equivalently,

$$
c_{2} \leqq c_{0} \vee c_{1} .
$$

The lattice $L$ is said to be arguesian provided, for any triangles $a$ and $b$ in $L$,

$$
p \leqq\left(a_{0} \wedge\left(a_{1} \vee m\right)\right) \vee b_{0} .
$$

We could have followed more closely the geometric theory and called a lattice arguesian provided it satisfies the condition $D(L)$ : Any two triangles in $L$ that are centrally perspective are also axially perspective. However, unlike this implication, the above definition is obviously equivalent to an identity. In any case, the two are known to be equivalent in the case of projective spaces, and in $B$. Jónsson and G. S. Monk [12], Theorem 3.4, it is shown that every arguesian lattice satisfies the condition $D(L)$. We shall now further justify the terminology by proving the converse of that assertion.

Lemma 1. For any modular lattice $L$, the following conditions are equivalent:

(i) $L$ is arguesian.

(ii) Any two triangles in $L$ that are centrally perspective are also axially perspective.

(iii) For any triangles $a=\left\langle a_{0}, a_{1}, a_{2}\right\rangle$ and $b=\left\langle b_{0}, b_{1}, b_{2}\right\rangle$ in $L$ that are centrally perspective, if $a_{0} \wedge a_{1} \leqq a_{2}$ and $b_{0} \wedge b_{1} \leqq b_{2}$, and if the center of perspectivity, $p$, satisfies the conditions

$$
a_{i} \vee p=b_{i} \vee p=a_{i} \vee b_{i} \quad \text { for } \quad i=0,1,2,
$$

then $a$ and $b$ are axially perspective. 
Proof. Since (i) is known to imply (ii), and (iii) is but a special case of (ii), we need only show that (iii) implies (i). Thus, assuming (iii), we consider two triangle $a$ and $b$ in $L$, and using the notation introduced earlier we wish to prove that

$$
p \leqq\left(a_{0} \wedge\left(a_{1} \vee m\right)\right) \vee b_{0} .
$$

We first reduce the problem to the special case in which

$$
p \vee a_{i}=p \vee b_{i}=a_{i} \vee b_{i} \text { for } i=0,1,2 \text {. }
$$

Assuming that (1) holds under this additional hypothesis, we consider two arbitrary triangles $a$ and $b$ in $L$, and we let

$$
a_{i}^{\prime}=a_{i} \wedge\left(b_{i} \vee p\right), b_{i}^{\prime}=b_{i} \wedge\left(a_{i} \vee p\right) \text { for } i=0,1,2 \text {. }
$$

Then

$$
p \vee a_{i}^{\prime}=p \vee b_{i}^{\prime}=a_{i}^{\prime} \vee b_{i}^{\iota} \text { for } i=0,1,2,
$$

whence it readily follows that

$$
p=\left(a_{0}^{\prime} \vee b_{0}^{\prime}\right) \wedge\left(a_{1}^{\prime} \vee b_{1}^{\prime}\right) \wedge\left(a_{2}^{\prime} \vee b_{2}^{\prime}\right) .
$$

Defining $c_{i}^{\prime}$ and $m^{\prime}$ in the same manner as $c_{i}$ and $m$ were defined, we therefore have

$$
p \leqq\left(a_{0}^{\prime} \wedge\left(a_{1}^{\prime} \vee m^{\prime}\right)\right) \vee b_{0}^{\prime} .
$$

Inasmuch as $a_{i}^{\prime} \leqq a_{i}, b_{i}^{\prime} \leqq b_{i}$, and $m^{\prime} \leqq m$, it follows that (1) holds.

We henceforth assume that (2) holds, and we shall prove that in this case $a_{0} \leqq a_{1} \vee m$, whence (1) certainly follows. Let

$$
q=\left(a_{0} \vee b_{0}\right) \wedge\left(a_{1} \vee b_{1}\right) \text {. }
$$

Then

$$
a_{0} \vee q=\left(a_{0} \vee b_{0}\right) \wedge\left(a_{0} \vee a_{1} \vee b_{1}\right) .
$$

Since $p \leqq a_{1} \vee b_{1}$ and, by (2), $b_{0} \leqq a_{0} \vee p$, this yields

$$
a_{0} \vee q=a_{0} \vee b_{0} \text {. }
$$

Similarly,

$$
b_{0} \vee q=a_{0} \vee b_{0}, a_{1} \vee q=b_{1} \vee q=a_{1} \vee b_{1} .
$$

Furthermore

$$
\begin{aligned}
& q=\left(a_{0} \vee p\right) \wedge\left(a_{1} \vee b_{1}\right)=p \vee\left(a_{0} \wedge\left(a_{1} \vee b_{1}\right)\right), \\
& q=\left(b_{0} \vee p\right) \wedge\left(a_{1} \vee b_{1}\right)=p \vee\left(b_{0} \wedge\left(a_{1} \vee b_{1}\right)\right) .
\end{aligned}
$$

Therefore, if we let 


$$
a_{2}^{*}=a_{2} \vee\left(a_{0} \wedge a_{1}\right), \quad b_{2}^{*}=b_{2} \vee\left(b_{0} \wedge\left(a_{1} \vee b_{1}\right)\right),
$$

then

$$
\begin{array}{r}
a_{2}^{*} \vee b_{2}^{*}=a_{2}^{*} \vee q=b_{2}^{*} \vee q, \\
a_{0} \wedge a_{1} \leqq a_{2}^{*}, \quad b_{0} \wedge b_{1} \leqq b_{2}^{*},
\end{array}
$$

and we can therefore apply (iii) to the triangles $\left\langle a_{0}, a_{1}, a_{2}^{*}\right\rangle$ and $\left\langle b_{0}, b_{1}, b_{2}^{*}\right\rangle$. Letting

$$
c_{0}^{*}=\left(a_{1} \vee a_{2}^{*}\right) \wedge\left(b_{1} \vee b_{2}^{*}\right), \quad c_{1}^{*}=\left(a_{2}^{*} \vee a_{0}\right) \wedge\left(b_{2}^{*} \vee b_{0}\right),
$$

we thus have

$$
c_{2} \leqq c_{0}^{*} \vee c_{1}^{*} \text {. }
$$

We now compute

$$
\begin{aligned}
c_{0}^{*} & =\left(a_{1} \vee a_{2}\right) \wedge\left(b_{1} \vee b_{2} \vee\left(b_{0} \wedge\left(a_{1} \vee b_{1}\right)\right)\right) \\
& =\left(a_{1} \vee a_{2}\right) \wedge\left(b_{2} \vee\left(\left(b_{0} \vee b_{1}\right) \vee\left(a_{1} \vee b_{1}\right)\right)\right) \\
& =\left(a_{1} \vee a_{2}\right) \wedge\left(b_{1} \vee b_{2} \vee\left(a_{1} \wedge\left(b_{0} \vee b_{1}\right)\right)\right) \\
& =\left(\left(a_{1} \vee a_{2}\right) \wedge\left(b_{1} \vee b_{2}\right)\right) \vee\left(a_{1} \wedge\left(b_{0} \vee b_{1}\right)\right) \\
& =c_{0} \vee\left(a_{1} \wedge\left(b_{0} \vee b_{1}\right)\right) .
\end{aligned}
$$

On the other hand it is easy to see that $c_{1}^{*}=c_{1}$. Thus

$$
\begin{aligned}
c_{2} & \leqq c_{0} \vee c_{1} \vee\left(a_{1} \wedge\left(b_{0} \vee b_{1}\right)\right), \\
c_{2} & =\left(c_{2} \wedge\left(c_{0} \vee c_{1}\right)\right) \vee\left(a_{1} \wedge\left(b_{0} \vee b_{1}\right)\right)=m \vee\left(a_{1} \wedge\left(b_{0} \vee b_{1}\right)\right), \\
a_{1} \vee m & =a_{1} \vee c_{2}=a_{0} \vee a_{1} \geqq a_{0},
\end{aligned}
$$

and the proof of Lemma 1 is complete.

Lemma 2. Suppose $L$ is a modular lattice, $u, v \in L$, and $v \leqq u$. If there exist elements $s, t \in L$ such that

$$
u \wedge s=u \wedge t=s \wedge t=v, \quad u \vee s=u \vee t=s \vee t,
$$

then the interval $[v, u]$ is an arguesian lattice.

Proof. Suppose the triangles $a=\left\langle a_{0}, a_{1}, a_{2}\right\rangle$ and $b=\left\langle b_{0}, b_{1}, b_{2}\right\rangle$ in $[v, u]$ satisfy the conditions of (iii) in Lemma 1 . Let $p, c_{0}, c_{1}$, and $c_{2}$ be defined as usual, and let

$$
\begin{aligned}
q & =s \wedge(p \vee t), \quad r=t \wedge(p \vee s), \\
d_{i} & =\left(a_{i} \vee q\right) \wedge\left(b_{i} \vee r\right) \text { for } i=0,1,2 .
\end{aligned}
$$

(See Figure 4.) Then 


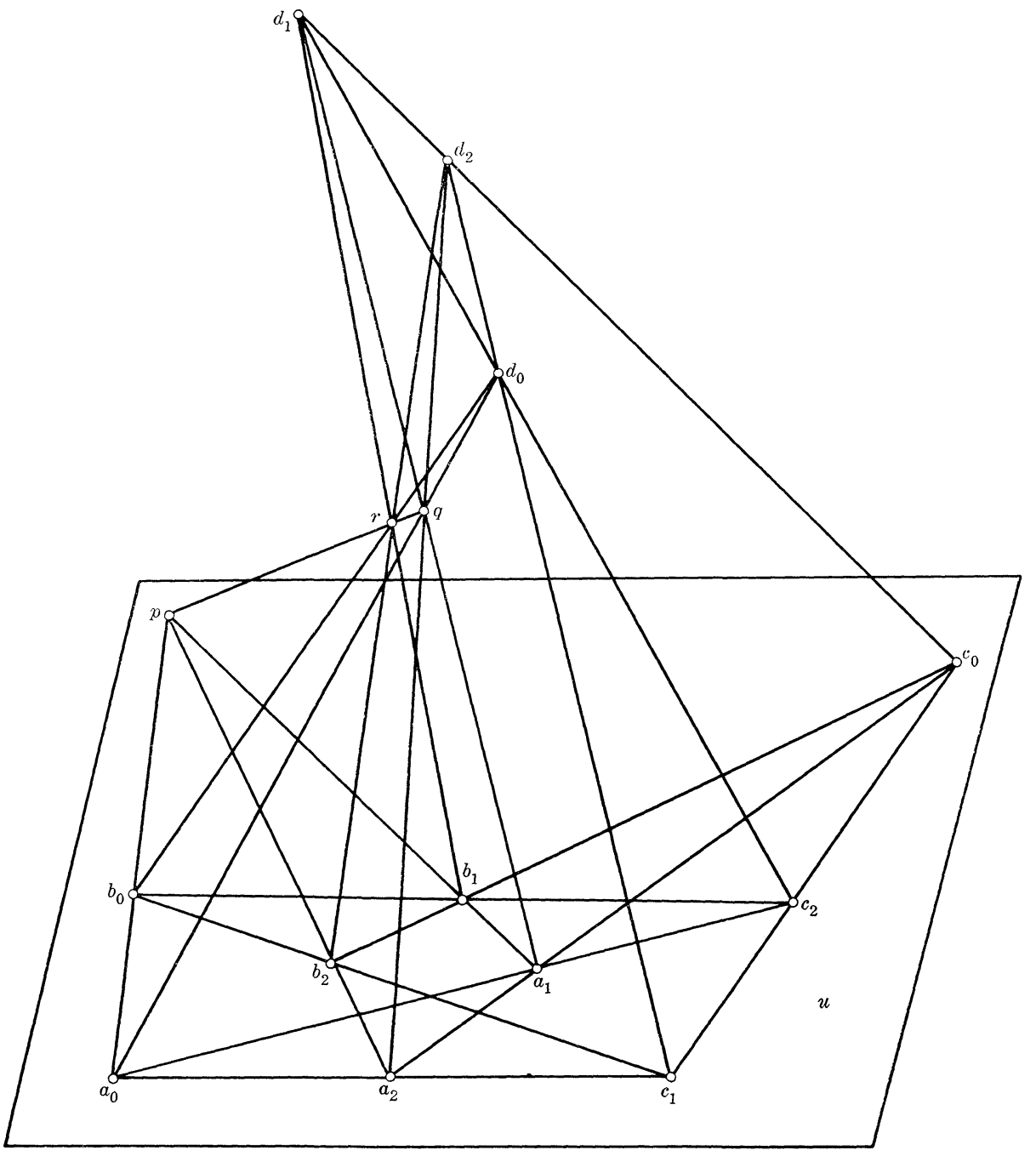

FIGURE 4.

$$
\begin{aligned}
p \vee q & =q \vee r=r \vee p=(p \vee s) \wedge(p \vee t), \\
p \wedge q & =q \wedge r=r \wedge p=v, \\
a_{i} \vee d_{i} & =\left(a_{i} \vee q\right) \wedge\left(a_{i} \vee b_{i} \vee r\right)=a_{i} \vee q,
\end{aligned}
$$

because $p \leqq a_{i} \vee b_{i}$ and $q \leqq p \vee r$. Also,

$$
d_{i} \vee q=\left(a_{i} \vee q\right) \wedge\left(b_{i} \vee q \vee r\right)=a_{i} \vee q,
$$

because $p \leqq q \vee r$ and $\alpha_{i} \leqq b_{i} \vee p$.

Observe that for any elements $x, y \in[v, u]$, the sets $\{x, y, q\}$ and $\{x, y, r\}$ generate a distributive lattice, because

$$
(x \vee y) \wedge q \leqq u \wedge q=v=(x \wedge q) \vee(y \vee q),
$$


and similarly with $q$ replaced by $r$. In particular, it follows from this that

$$
(x \vee q) \wedge y=x \wedge y, \quad(x \vee q) \wedge(y \vee q)=(x \wedge y) \vee q .
$$

These observations will be used several times below. We could use them at this point to prove that the triangle $d=\left\langle d_{0}, d_{1}, d_{2}\right\rangle$ is centrally perspective with each of the triangles $a$ and $b$, but no use will be made of that fact.

We shall presently need the formula

$$
a_{0} \wedge\left(b_{0} \vee b_{1}\right) \leqq d_{0} \vee d_{1}
$$

To verify this inequality we compute

$$
\begin{aligned}
d_{0} \vee d_{1} & \geqq\left(\left(a_{0} \vee q\right) \wedge\left(b_{0} \vee r\right)\right) \vee\left(q \wedge\left(b_{1} \vee r\right)\right) \\
& =\left(a_{0} \vee q\right) \wedge\left(b_{0} \vee r \vee\left(q \wedge\left(b_{1} \vee r\right)\right)\right) \\
& =\left(a_{0} \vee q\right) \wedge\left(b_{0} \vee\left((q \vee r) \wedge\left(b_{1} \vee r\right)\right)\right) \\
& =\left(a_{0} \vee q\right) \wedge\left(b_{0} \vee r \vee\left(b_{1} \wedge(q \vee r)\right)\right) \\
& \geqq a_{0} \wedge\left(b_{0} \vee\left(b_{1} \wedge p\right)\right)=a_{0} \wedge\left(b_{0} \vee\left(b_{1} \wedge\left(a_{0} \vee b_{0}\right)\right)\right) \\
& =a_{0} \wedge\left(b_{0} \vee b_{1}\right) .
\end{aligned}
$$

We now wish to prove the inequalities

$$
\begin{aligned}
& c_{2} \leqq d_{0} \vee d_{1}, \\
& d_{1} \leqq c_{0} \vee d_{2}, \quad d_{0} \leqq c_{1} \vee d_{2} .
\end{aligned}
$$

For the proof of (4) we first note that

$$
\left(a_{0} \vee q\right) \wedge\left(b_{0} \vee b_{1} \vee r\right) \leqq d_{0} \vee d_{1} .
$$

In fact,

$$
\begin{aligned}
\left(a_{0} \vee q\right) \wedge\left(b_{0} \vee b_{1} \vee r\right) & \leqq\left(a_{0} \vee p \vee q\right) \wedge\left(b_{0} \vee b_{1} \vee r\right) \\
& =\left(\left(a_{0} \vee p \vee q\right) \wedge\left(b_{0} \vee b_{1}\right)\right) \vee r \\
& =\left(\left(a_{0} \vee b_{0}\right) \wedge\left(b_{0} \vee b_{1}\right)\right) \vee r \\
& =\left(a_{0} \wedge\left(b_{0} \vee b_{1}\right)\right) \vee b_{0} \vee r, \\
\left(a_{0} \vee q\right) \wedge\left(b_{0} \vee b_{1} \vee r\right) & \leqq\left(a_{0} \vee q\right) \wedge\left(\left(a_{0} \wedge\left(b_{0} \vee b_{1}\right)\right) \vee b_{0} \vee r\right) \\
& =\left(a_{0} \wedge\left(b_{0} \vee b_{1}\right)\right) \vee\left(\left(a_{0} \vee q\right) \wedge\left(b_{0} \vee r\right)\right) \\
& =\left(a_{0} \wedge\left(b_{0} \vee b_{1}\right)\right) \vee d_{1} .
\end{aligned}
$$

In view of (3), this implies (6). Using (6) we compute

$$
\begin{aligned}
d_{0} \vee d_{1} & \geqq\left(\left(a_{0} \vee q\right) \wedge\left(b_{0} \vee b_{1} \vee r\right)\right) \vee d_{1} \\
& =\left(a_{0} \vee q \vee d_{1}\right) \wedge\left(b_{0} \vee b_{1} \vee r\right) \\
& =\left(a_{0} \vee a_{1} \vee q\right) \wedge\left(b_{0} \vee b_{1} \vee r\right) \geqq c_{2} .
\end{aligned}
$$


To prove the first inequality in (5) we first show that

$$
\left(a_{1} \vee a_{2} \vee q\right) \wedge\left(b_{2} \vee r\right) \leqq c_{0} \vee d_{2} \text {. }
$$

In fact,

$$
\begin{aligned}
\left(a_{1} \vee a_{2} \vee q\right) \wedge\left(b_{2} \vee r\right) & \leqq\left(a_{1} \vee a_{2} \vee q\right) \wedge\left(b_{2} \vee p \vee r\right) \\
& \leqq q \vee\left(\left(a_{1} \vee a_{2}\right) \wedge\left(b_{2} \vee p \vee r\right)\right) \\
& =q \vee\left(\left(a_{1} \vee a_{2}\right) \wedge\left(a_{2} \vee b_{2}\right)\right) \\
& =q \vee a_{2} \vee\left(b_{2} \wedge\left(a_{1} \vee a_{2}\right)\right) \\
\left(a_{1} \vee a_{2} \vee q\right) \wedge\left(b_{2} \vee r\right) & \leqq\left(q \vee a_{2} \vee\left(b_{2} \wedge\left(a_{1} \vee a_{2}\right)\right)\right) \wedge\left(b_{2} \vee r\right) \\
& =\left(\left(a_{2} \vee q\right) \wedge\left(b_{2} \vee r\right)\right) \vee\left(b_{2} \wedge\left(a_{1} \vee a_{2}\right)\right) \\
& =d_{2} \vee\left(b_{2} \wedge\left(a_{1} \vee a_{2}\right)\right) \leqq c_{0} \vee d_{2} .
\end{aligned}
$$

With the aid of (7) we find that

$$
\begin{aligned}
c_{0} \vee d_{2} & \geqq\left(\left(a_{1} \vee a_{2} \vee q\right) \wedge\left(b_{2} \vee r\right)\right) \vee c_{0} \\
& =\left(a_{1} \vee a_{2} \vee q\right) \wedge\left(b_{2} \vee c_{0} \vee r\right) \\
& =\left(a_{1} \vee a_{2} \vee q\right) \wedge\left(b_{1} \vee b_{2} \vee r\right) \geqq d_{1} .
\end{aligned}
$$

The first formula in (5) therefore holds, and the second follows by symmetry.

Using (4) and (5) we find that

$$
\begin{aligned}
c_{2} \leqq\left(d_{0} \vee d_{1}\right) \wedge u & \leqq\left(c_{0} \vee c_{1} \vee d_{2}\right) \wedge u \\
& =c_{0} \vee c_{1} \vee\left(d_{2} \wedge u\right) .
\end{aligned}
$$

Since $d_{2} \wedge u=\left(a_{2} \vee q\right) \wedge u \wedge\left(b_{2} \vee r\right) \wedge u=a_{2} \wedge b_{2}$, we conclude that

$$
c_{2} \leqq c_{0} \vee c_{1} \text {. }
$$

The proof of Lemma 2 is now complete.

LEMma 3. If $\boldsymbol{K}$ is any equational class of lattices, and if $\operatorname{Amal}(\boldsymbol{K})$ contains a distributive lattice with more than one element, then the two-element chain belongs to Amal $(\boldsymbol{K})$.

Proof. Let $C_{2}=\{0,1\}$ be the two-element chain, let $B$ be a distributive lattice, and let $C \in \boldsymbol{K}$. Let $f_{0}: C_{2} \rightarrow B, f_{1}: C_{2} \rightarrow C$ be oneto-one homomorphisms. We show that this amalgamation can be effected in $K$. Since $B$ is distributive there is a retraction $\rho: B \rightarrow C_{2}$ such that $f_{0} o=1_{C_{2}}$. Given any $x, y \in C, x \neq y$, define $g_{1}: C \rightarrow C$ as the identity and define $g_{0}: B \rightarrow C$ by $g_{0}=\rho f_{1}$. Then $f_{0} g_{0}=f_{1} g_{1}$ and $x g_{1} \neq y g_{1}$. For any $x, y \in B, x \neq y$, there is a homomorphism $\bar{h}: B \rightarrow C_{2}$ such that $x \bar{h} \neq y \bar{h}$ since $B$ is distributive. Define $h_{0}: B \rightarrow C$ by 
$h_{0}=\bar{h} f_{1}$. If $0 f_{0} \bar{h}=1 f_{0} \bar{h}$ define $h_{1}: C \rightarrow C$ by $z h_{1}=0 f_{0} h_{0}$. If $0 f_{0} \bar{h} \neq$ $1 f_{0} \bar{h}$ define $h_{1}: C \rightarrow C$ as the identity. In either case $f_{0} h_{0}=f_{1} h_{1}$ and $x h_{0} \neq y h_{0}$. It then follows (see Theorem 13.18 of [5]) that there is a lattice $D \in \boldsymbol{K}$ and embeddings $g_{0}: B \rightarrow D, g_{1}: C \rightarrow D$ such that $f_{0} g_{0}=$ $f_{1} g_{1}$.

Now suppose $B, C \in \boldsymbol{K}$, and suppose $f_{0}: C_{2} \rightarrow B$ and $f_{1}: C_{2} \rightarrow C$ are embeddings. By hypothesis there exists a distributive lattice $A \in \operatorname{Amal}(K)$ with more than one element. Choosing an embedding $\varphi: C_{2} \rightarrow A$, we effect three amalgamations as indicated in Figure 5, using Lemma 3

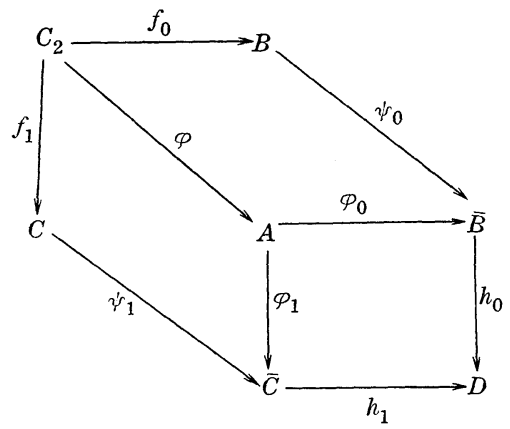

FIGURE 5 .

twice to obtain the pairs $\varphi_{0}, \psi_{0}$ and $\varphi_{1}, \psi_{1}$, and finally using the fact that $A$ is in the Amalgamation Class of $\boldsymbol{K}$ to obtain $h_{0}$ and $h_{1}$. Then $g_{0}=\psi_{0} h_{0}$ and $g_{1}=\psi_{1} h_{1}$ are embeddings of $B$ and $C$ into $D$ with $f_{0} g_{0}=f_{1} g_{1}$. Thus $C_{2} \in \operatorname{Amal}(\boldsymbol{K})$.

Now we are ready to prove Theorem 2. Assume that $K$ is an equational class of modular lattices and that Amal $(\boldsymbol{K})$ contains a distributive lattice with more than one element. We have to prove that every $L \in \boldsymbol{K}$ is arguesian. It is sufficient to prove this for finitely generated $L$, and so $L$ has a 0 and a 1 . Since every distributive lattice is arguesian we may assume that $\boldsymbol{K}$ contains a nondistributive lattice, and hence it contains the diamond. Let $C_{2}=$ $\{0,1\}$ be the two-element chain, $f_{0}$ the embedding of $C_{2}$ into $L$ defined by

$$
0 f_{0}=0 \text { and } 1 f_{0}=1 \text {. }
$$

Let $f_{1}$ embed $C_{2}$ into the diamond (see Figure 2) by

$$
0 f_{1}=o \text { and } 1 f_{1}=a \text {. }
$$

Since $C_{2} \in \operatorname{Amal}(\boldsymbol{K})$, there exist $C \in \boldsymbol{K}$ and embeddings $g_{0}, g_{1}$ into $C$ of $L$ and of the diamond respectively, such that $f_{0} g_{0}=f_{1} g_{1}$. By Lemma 2, the interval $\left[\log _{0}, a g_{0}\right]$ is arguesian. Since $L$ is isomorphic to $L g_{0}$ 
and $L g_{0} \subseteq\left[o g_{1}, a g_{1}\right]$, we conclude that $L$ is arguesian, completing the proof of the second statement of Theorem 2 .

If $P$ is a projective plane that does not satisfy Desargues' theorem, then $\mathscr{L}(P)$ is not arguesian, hence the second statement in Theorem 2 implies the first one.

4. Concluding remarks. Lemma 3 above provides some information about the amalgamation classes of nonmodular equational classes. The following simple theorem, essentially obtained by A. Day by using and extending earlier ideas due to $\mathrm{S}$. Comer and S. Fajtlowicz, contains further information.

THeOREM 3. Let $\boldsymbol{K}$ be an equational class of lattices generated by a finite nondistributive lattice. Then $\boldsymbol{K}$ does not have the Amalgamation Property. In fact, the two-element chain does not belong to $\operatorname{Amal}(\boldsymbol{K})$.

Proof. By a result of B. Jónsson [10] there exists an integer $n$ such that every subdirectly irreducible lattice in $\boldsymbol{K}$ has not more than $n$ elements.

Now assume that $C_{2} \in \operatorname{Amal}(\boldsymbol{K})$. Since $\boldsymbol{K}$ is nondistributive $\boldsymbol{K}$ contains either the diamond (Figure 2) or $N_{5}$ (Figure 6). In the first case $K$ has to contain all the lattices $A_{k}$ of Figure 7. Indeed, $A_{1}$ is the diamond, so $A_{1} \in \boldsymbol{K}$ by assumption. If $A_{k} \in \boldsymbol{K}$, then consider the embedding $f_{0}$ of $C_{2}$ into the diamond defined by $0 f_{0}=a$,

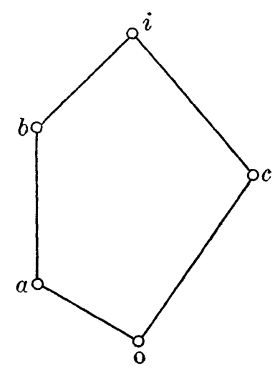

FIGURE 6.

$1 f_{0}=i$ and the embedding $f_{1}: C_{2} \rightarrow A_{k}$ given by $0 f_{1}=x, 1 f_{1}=i$. Let $g_{0}, g_{1}, L$ amalgamate this in $\boldsymbol{K}$. Then Image $\left(f_{0} g_{0}\right) \cup$ Image $\left(f_{1} g_{1}\right)$ is a sublattice of $L$ isomorphic to $A_{k+1}$. Hence $A_{k} \in \boldsymbol{K}$ for all $k$. This contradicts the fact that $\left|A_{k}\right|>n$ for some $k$.

In the second case (i.e., $N_{5} \in \boldsymbol{K}$ ) let $A$ be any finite subdirectly irreducible lattice and consider $f_{0}: C_{2} \rightarrow A, f_{1}: C_{2} \rightarrow N_{5}$ defined by

$$
0 f_{0}=0,1 f_{0}=1 ; \quad 0 f_{1}=a, 1 f_{1}=b .
$$




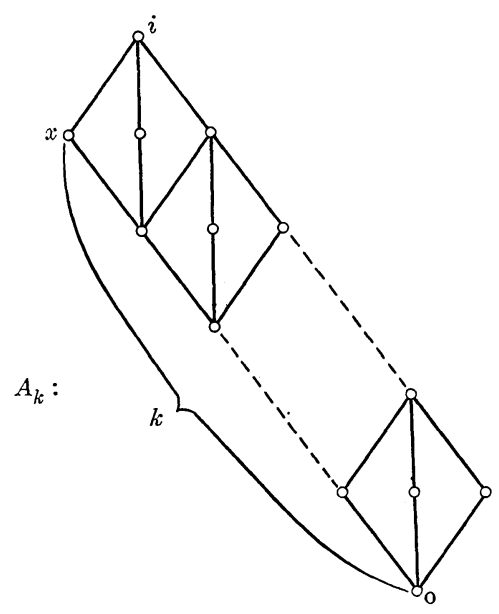

FIgURe 7.

Let $g_{0}, g_{1}, B$ amalgamate these in $\boldsymbol{K}$. The Image $\left(f_{0} g_{0}\right) \cup$ Image $\left(f_{1} g_{1}\right)=$ $A_{N}$ is a sublattice of $B$; hence the $A_{N}$ of Figure 8 is in $\boldsymbol{K}$. Obviously

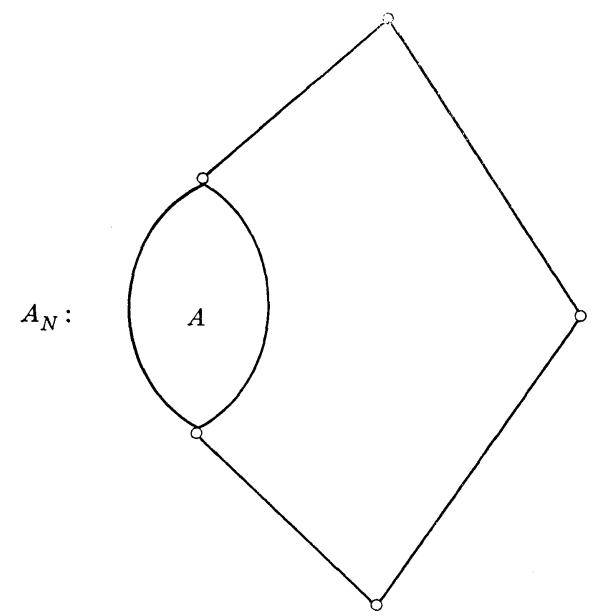

FIGURE 8.

$A_{N}$ is subdirectly irreducible and $\left|A_{N}\right|=|A|+3$. Repeating this sufficiently many times we construct a subdirectly irreducible lattice in $\boldsymbol{K}$ with more than $n$ elements. This contradiction completes the proof of this theorem.

The results of this paper show that equational classes of lattices having the Amalgamation Property are hard to find.

We do not know of any lattice with more than one element that belongs to Amal $(\boldsymbol{M})$. Is there any?

For a division ring $D$, let $\boldsymbol{K}(D)$ denote the class of all lattices 
embeddable in some $\mathscr{L}(V, D)$. E. Fried [2[ has shown that all finite distributive lattices and also the diamond belong to Amal $(K(D))$.

Theorem 2 is much weaker than Theorem 1 in that it only gives a necessary condition for $C_{2} \in \operatorname{Amal}(\boldsymbol{K})$, where $\boldsymbol{K}$ is an equational class of modular lattices. We do not know whether such classes exist excepting of course the class of distributive lattices and the trivial equational class.

Our information about amalgamation classes in general is still very limited. The following two observations are valid for any equational class $\boldsymbol{K}$ of algebras:

Suppose $A^{\prime} \in \operatorname{Amal}(\boldsymbol{K})$, and $\varphi: A \rightarrow A^{\prime}$ is an embedding. If, for every $B \in \boldsymbol{K}$, and every embedding $\psi: A \rightarrow B$, the amalgam $\langle A, \varphi$, $\left.A^{\prime}, \psi, B\right\rangle$ can be amalgamated in $\boldsymbol{K}$, then $A \in \operatorname{Amal}(\boldsymbol{K})$.

If $A \in \boldsymbol{K}$ and $A$ is an absolute retract in $\boldsymbol{K}$, then $A \in \operatorname{Amal}(\boldsymbol{K})$.

A proof of the first observation is essentially contained in the proof of Lemma 3 in $\S 3$. To prove the second, suppose $B, C \in \boldsymbol{K}$, and suppose $f_{0}: A \rightarrow B$ and $f_{1}: A \rightarrow C$ are embeddings. Since $A$ is an absolute retract in $K$, there exist onto homomorphisms $\varphi_{0}: B \rightarrow A$ and $\varphi_{1}: C \rightarrow A$ such that $b \varphi_{0} f_{0}=b$ for all $b \in B$ and $c \varphi_{1} f_{1}=c$ for all $c \in C$. Let $D=B \times C$, and for $b \in B$ and $c \in C$ let

$$
b g_{0}=\left\langle b, b \varphi_{0} f_{1}\right\rangle, \quad c g_{1}=\left\langle c \varphi_{1} f_{0}, c\right\rangle \text {. }
$$

Then $g_{0}: B \rightarrow D$ and $g_{1}: C \rightarrow D$ are embeddings, and $f_{0} g_{0}=f_{1} g_{1}$.

From these two observations one readily obtains:

If $A \times A^{\prime} \in \operatorname{Amal}(\boldsymbol{K})$, and if $A^{\prime}$ has a one-element subalgebra, then $A \in \operatorname{Amal}(\boldsymbol{K})$.

If some reduced power of $A$ belongs to Amal $(\boldsymbol{K})$, then $A \in$ Amal $(\boldsymbol{K})$.

If $A \in \boldsymbol{K}, A$ is subdirectly irreducible and of finite order $n$, and $\boldsymbol{K}$ has no subdirectly irreducible member whose order is larger than $n$, then $A \in \operatorname{Amal}(\boldsymbol{K})$.

For the case when $K$ is an equational class of lattices we also have:

If the lattice of all ideals of $L$ belongs to Amal $(\boldsymbol{K})$, then $L \in \operatorname{Amal}(\boldsymbol{K})$.

If an ordered sum of two or more lattices belongs to Amal $(\boldsymbol{K})$, then each summand belongs to $\operatorname{Amal}(\boldsymbol{K})$.

If $\boldsymbol{K}$ is generated by a finite, subdirectly irreducible lattice $L$, then $L \in \operatorname{Amal}(\boldsymbol{K})$.

For certain equational classes $\boldsymbol{K}$ of modular lattices, specifically those generated by lattices of length two, the finite members of Amal $(\boldsymbol{K})$ are determined in E. Fried, G. Grätzer, and H. Lakser [3]. 
Remark added in proof. It has been proved by M. Yashuhara that if $\boldsymbol{K}$ is an equational class, then every member of $\boldsymbol{K}$ can be extended to a member of Amal $(\boldsymbol{K})$. This shows in particular that Amal $(\boldsymbol{M})$ is very big. However, no lattice with more than one element is known to belong to $\operatorname{Amal}(\boldsymbol{M})$.

\section{REFERENCES}

1. R. P. Dilworth and M. Hall, The imbedding problem for modular lattices, Ann. of Math., 45 (1944), 450-456.

2. E. Fried, The amalgamation class of certain classes of projective geometries, to appear.

3. E. Fried, G. Grätzer, and H. Lakser, Amalgamation and weak injectives in the equational class of modular lattices $\boldsymbol{M}_{n}$, to appear.

4. O. Frink, Complemented modular lattices and projective spaces of infinite dimension, Trans. Amer. Math. Soc., 60 (1946), 452-467.

5. G. Grätzer, Lattice Theory: First Concepts and Distributive Lattices, W. H. Freeman, San Francisco, Calif., 1971.

6. G. Grätzer and H. Lakser, The structure of pseudocomplemented distributive lattices. II: Congruence extension and amalgamation, Trans. Amer. Math. Soc., 156 (1971), 343-358.

7. - Modular lattices and the amalgamation preperty, Notices Amer. Math, Soc., 18 (1971), 618.

8. B. Jónsson, Extensions of Relational Structures. Theory of Models, Proc. 1963 Int. Symp. Berkeley, North-Holland, 1965.

9. — Modular lattices and Desargues' Theorem, Math. Scand., 2 (1954), 29a314.

10. - Algebras whose congruence lattices are distributive, Math. Scand., 21 (1967), $110-121$.

11. The amalgamation property in varieties of modular lattices, Notices Amer. Math. Soc., 18 (1971), 400.

12. B. Jónsson and G. S. Monk, Representations of primary Arguesian lattices, Pacific. J. Math., 29 (1969), 95-140.

Received November 19, 1971. The research of the first and third authors was supported by the National Research Council of Canada. The work of the second author was supported in part by the National Science Foundation. Some of the results were announced in the Notices of the American Mathematical Society.

The University of MANITOBA

AND

VANDERBILT UNIVERSITY 


\section{PACIFIC JOURNAL OF MATHEMATICS}

\section{EDITORS}

\author{
H. SAMELson \\ Stanford University \\ Stanford, California 94305 \\ C. R. HоввY \\ University of Washington \\ Seattle, Washington 98105
}

J. DugundJI

Department of Mathematics

University of Southern California

Los Angeles, California 90007

RICHARD ARENS

University of California

Los Angeles, California 90024

\section{ASSOCIATE EDITORS}
E. F. BeCKENBACH
B. H. NEUMANN
F. WOLF
K. YoshidA

\section{SUPPORTING INSTITUTIONS}

UNIVERSITY OF BRITISH COLUMBIA

CALIFORNIA INSTITUTE OF TECHNOLOGY

UNIVERSITY OF CALIFORNIA

MONTANA STATE UNIVERSITY

UNIVERSITY OF NEVADA

NEW MEXICO STATE UNIVERSITY

OREGON STATE UNIVERSITY

UNIVERSITY OF OREGON

OSAKA UNIVERSITY

\author{
UNIVERSITY OF SOUTHERN CALIFORNIA \\ STANFORD UNIVERSITY \\ UNIVERSITY OF TOKYO \\ UNIVERSITY OF UTAH \\ WASHINGTON STATE UNIVERSITY \\ UNIVERSITY OF WASHINGTON

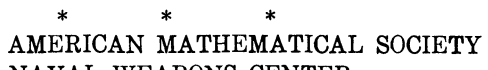 \\ NAVAL WEAPONS CENTER
}

The Supporting Institutions listed above contribute to the cost of publication of this Journal, but they are not owners or publishers and have no responsibility for its content or policies.

Mathematical papers intended for publication in the Pacific Journal of Mathematics should be in typed form or offset-reproduced, (not dittoed), double spaced with large margins. Underline Greek letters in red, German in green, and script in blue. The first paragraph or two must be capable of being used separately as a synopsis of the entire paper. The editorial "we" must not be used in the synopsis, and items of the bibliography should not be cited there unless absolutely necessary, in which case they must be identified by author and Journal, rather than by item number. Manuscripts, in duplicate if possible, may be sent to any one of the four editors. Please classify according to the scheme of Math. Rev. Index to Vol. 39. All other communications to the editors should be addressed to the managing editor, Richard Arens, University of California, Los Angeles, California, 90024.

50 reprints are provided free for each article; additional copies may be obtained at cost in multiples of 50 .

The Pacific Journal of Mathematics is issued monthly as of January 1966. Regular subscription rate: $\$ 48.00$ a year (6 Vols., 12 issues). Special rate: $\$ 24.00$ a year to individual members of supporting institutions.

Subscriptions, orders for back numbers, and changes of address should be sent to Pacific Journal of Mathematics, 103 Highland Boulevard, Berkeley, California, 94708.

PUBLISHED BY PACIFIC JOURNAL OF MATHEMATICS, A NON-PROFIT CORPORATION

Printed at Kokusai Bunken Insatsusha (International Academic Printing Co., Ltd.), 270, 3-chome Totsuka-cho, Shinjuku-ku, Tokyo 160, Japan. 


\section{Pacific Journal of Mathematics}

\section{Vol. 45, No. $2 \quad$ October, 1973}

Kenneth Paul Baclawski and Kenneth Kapp, Induced topologies for quasigroups and loops ............................................. 393

D. G. Bourgin, Fixed point and $\min -\max$ theorems $\ldots \ldots \ldots \ldots \ldots \ldots \ldots$

J. L. Brenner, Zolotarev's theorem on the Legendre symbol ............... 413

Jospeh Atkins Childress, Jr., Restricting isotopies of spheres .............. 415

John Edward Coury, Some results on lacunary Walsh series ................ 419

James B. Derr and N. P. Mukherjee, Generalized Sylow tower groups. II . . . . . . 427

Paul Frazier Duvall, Jr., Peter Fletcher and Robert Allen McCoy, Isotopy Galois

spaces .......................................... 435

Mary Rodriguez Embry, Strictly cyclic operator algebras on a Banach space ... 443

Abi (Abiadbollah) Fattahi, On generalizations of Sylow tower groups ......... 453

Burton I. Fein and Murray M. Schacher, Maximal subfields of tensor products . . 479

Ervin Fried and J. Sichler, Homomorphisms of commutative rings with unit

element .......................................... 485

Kenneth R. Goodearl, Essential products of nonsingular rings ............. 493

George Grätzer, Bjarni Jónsson and H. Lakser, The amalgamation property in

equational classes of modular lattices ...........................

507

$\mathrm{H}$. Groemer, On some mean values associated with a randomly selected simplex

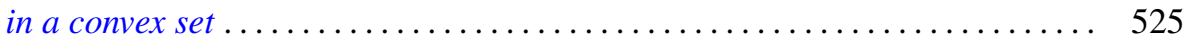

Marcel Herzog, Central 2-Sylow intersections .................... 535

Joel Saul Hillel, On the number of type-k translation-invariant groups ........ 539

Ronald Brian Kirk, A note on the Mackey topology for $\left(C^{b}(X)^{*}, C^{b}(X)\right) \ldots \ldots .543$

J. W. Lea, The peripherality of irreducible elements of lattice.............. 555

John Stewart Locker, Self-adjointness for multi-point differential operators ..... 561

Robert Patrick Martineau, Splitting of group representations ............... 571

Robert Massagli, On a new radical in a topological ring ................. 577

James Murdoch McPherson, Wild arcs in three-space. I. Families of Fox-Artin

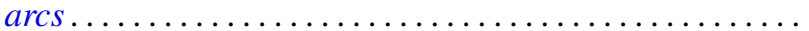

585

James Murdoch McPherson, Wild arcs in three-space. III. An invariant of

oriented local type for exceptional arcs . . . . . . . . . . . . ............ 599

Fred Richman, The constructive theory of countable abelian p-groups ........ 621

Edward Barry Saff and J. L. Walsh, On the convergence of rational functions

which interpolate in the roots of unity ..................

Harold Eugene Schlais, Non-aposyndesis and non-hereditary

decomposability..................................... 643

Mark Lawrence Teply, A class of divisible modules................... 653

Edward Joseph Tully, Jr., H-commutative semigroups in which each

homomorphism is uniquely determined by its kernel ................. 669

Garth William Warner, Jr., Zeta functions on the real general linear group ...... 681

Keith Yale, Cocyles with range $\{ \pm 1\} \ldots \ldots \ldots \ldots \ldots \ldots \ldots \ldots \ldots \ldots \ldots \ldots \ldots . \ldots \ldots$

Chi-Lin Yen, On the rest points of a nonlinear nonexpansive semigroup ........ 699 